PROCEEDINGS OF THE

AMERICAN MATHEMATICAL SOCIETY

Volume 125, Number 4, April 1997, Pages 1149-1152

S 0002-9939(97)03764-7

\title{
ON FUNCTIONS ARISING AS POTENTIALS ON SPACES OF HOMOGENEOUS TYPE
}

\author{
A. EDUARDO GATTO AND STEPHEN VÁGI
}

(Communicated by J. Marshall Ash)

\begin{abstract}
On a space of homogeneous type we consider functions $F$ in $L^{p}$, $1<p<\infty$, which are potentials of order $\alpha$ of $L^{p}$ functions. We show that these functions belong to the class of smooth functions $C^{p, \alpha}$ of Calderón-Scott. This result has applications to tangential convergence.
\end{abstract}

\section{INTRODUCTION}

This note is motivated by the results in [CDS] on boundary tangential convergence on spaces of homogeneous type. In that paper the authors showed that $C^{p, \alpha}$ smoothness of functions defined on a space of homogeneous type suffices for obtaining boundary tangential convergence of "convolutions" with approximate identities, $1<p \leq \frac{1}{\alpha}$, thus extending the results in [NRS]. It is shown in [CDS] that in the case of stratified nilpotent Lie groups the spaces of potentials, as defined in $[\mathrm{F}]$, are continuously embedded in $C^{p, \alpha}$ spaces.

Our purpose is to show that in a general space of homogeneous type $L^{p}$ functions that are potentials of order $\alpha$ of functions in $L^{p}$ are included in the classes $C^{p, \alpha}$, and that this can be proved by adapting a classical argument for sharp functions [S, p. 158].

\section{DEFINITIONS AND STATEMENT OF THE RESUltS}

In this paper $(X, \delta, \mu)$ is a space of homogeneous type as defined in $[\mathrm{CW}]$ or [MS] such that $\mu(X)=\infty$ and $\mu(\{x\})=0$ for all $x$ in $X$. As shown in [MS] we can assume without loss of generality that $(X, \delta, \mu)$ is a normal space of order $\gamma, 0<\gamma \leq 1$. This means that the quasidistance $\delta$ and the measure $\mu$ have the following two properties:

1. For all $r>0$ and all $x$ in $X$ the balls $B_{r}(x)=\{y \in X \mid \delta(x, y)<r\}$ satisfy

$$
c_{1} r \leq \mu\left(B_{r}(x)\right) \leq c_{2} r
$$

with positive constants $c_{1}$ and $c_{2}$ independent of $x$ and $r$.

2. There exists a number $\gamma, 0<\gamma \leq 1$, called the order of $(X, \delta, \mu)$ such that for all $x, x^{\prime}$ and $y$ in $X$ we have

$$
\left|\delta(x, y)-\delta\left(x^{\prime}, y\right)\right| \leq M \delta^{\gamma}\left(x, x^{\prime}\right)\left\{\delta(x, y)+\delta\left(x^{\prime}, y\right)\right\}^{1-\gamma}
$$

with a positive constant $M$ independent of $x, x^{\prime}$ and $y$.

Received by the editors October 25, 1995 and, in revised form, January 25, 1996.

1991 Mathematics Subject Classification. Primary 42C99, 26A33, 44A99; Secondary 31C15.

(C)1997 American Mathematical Society 
We shall need the following known Lemma [GV, Lemma II.3].

Lemma. Let $0<\alpha<1$. Then for all $x, x^{\prime}$ and $y$ in $X$ such that $\delta(x, y)>2 \kappa \delta\left(x, x^{\prime}\right)$

$$
\left|\delta^{\alpha-1}(x, y)-\delta^{\alpha-1}\left(x^{\prime}, y\right)\right| \leq K \delta^{\gamma}\left(x, x^{\prime}\right) \delta^{\alpha-\gamma-1}(x, y)
$$

where $\kappa$ is the constant of the "triangle" inequality of $\delta$, and $K$ is a constant independent of $x, x^{\prime}$ and $y$.

The (Riesz) potential of order $\alpha, 0<\alpha<\gamma$, of a function $f$ in $L^{p}$ with $1<p<\frac{1}{\alpha}$ is defined as in $[\mathrm{GV}]$ by

$$
I_{\alpha} f(x)=\int_{X} \frac{f(y)}{\delta^{1-\alpha}(x, y)} d \mu(y),
$$

and for $\frac{1}{\alpha} \leq p<\infty$ by

$$
\widetilde{I}_{\alpha} f(x)=\int_{X} f(y)\left[\frac{1}{\delta^{1-\alpha}(x, y)}-\frac{\psi_{u}(y)}{\delta^{1-\alpha}(u, y)}\right] d \mu(y)
$$

where $u$ is a fixed point in $X, \psi_{u}(y)$ is the characteristic function of the complement of the ball $B_{1}(u)$ when $p=\frac{1}{\alpha}$, and, for $\frac{1}{\alpha}<p, \psi_{u}(y)$ is identically 1 on $X$. It can be seen that $I_{\alpha} f(x)$ and $\widetilde{I}_{\alpha} f(x)$ converge absolutely for almost every $x$ in $X$ [GV].

The spaces $C^{p, \alpha}$ of smooth functions of Calderón-Scott [CS] were introduced on spaces of homogeneous type in [CDS]. For $f \in L^{p}, 1<p<\infty$, we consider the sharp functions

$$
f_{\alpha}^{\#}(x)=\sup _{x \in B} \frac{1}{\mu(B)^{1+\alpha}} \int_{B}\left|f(y)-m_{B}(f)\right| d \mu(y)
$$

where $m_{B}(f)=\frac{1}{\mu(B)} \int_{B} f(y) d \mu(y)$. The space $C^{p, \alpha}$ is the set of all functions $f \in L^{p}$ with $f_{\alpha}^{\#} \in L^{p}$ equipped with the norm $\|f\|_{C^{p, \alpha}}=\left\|f_{\alpha}^{\#}\right\|_{p}+\|f\|_{p}$, where \|\|$_{p}$ denotes the $L^{p}$-norm. The letter $c$ will denote a constant, not necessarily the same in different occurrences.

We now state the results.

Theorem 2.1. Let $0<\alpha<\gamma, 1<p<\frac{1}{\alpha}$ and $F \in L^{p}$ with $F=I_{\alpha}(f)$ and $f \in L^{p}$. Then $F \in C^{p, \alpha}$ and $\|F\|_{C^{p, \alpha}} \leq c\left(\|F\|_{p}+\|f\|_{p}\right)$, with a constant $c$ independent of $F$ and $f$.

Theorem 2.2. Let $0<\alpha<\gamma, \frac{1}{\alpha} \leq p<\infty$, and $F \in L^{p}$ with $F=\widetilde{I}_{\alpha}(f)+C_{F}$ where $f \in L^{p}$ and $C_{F}$ is a constant. Then $F \in C^{p, \alpha}$ and $\|F\|_{C^{p, \alpha}} \leq c\left(\|F\|_{p}+\|f\|_{p}\right)$, with $c$ independent of $F$ and $f$.

\section{Proof}

Proof of Theorem 2.1. Fix $x \in X$; we will estimate $F_{\alpha}^{\#}(x)$. Let $B=B_{r}\left(x_{0}\right)$ be a ball containing $x$ and $\widetilde{B}=B_{2 \kappa r}\left(x_{0}\right)$, where $\kappa$ is the constant of the "triangle" inequality of $\delta$, and, finally, let $\chi$ be the characteristic function of $\widetilde{B}$. Set $F=F_{1}+F_{2}$ with $F_{1}=I_{\alpha}(f \chi)$ and $F_{2}=I_{\alpha}(f(1-\chi))$. To estimate $\left(F_{1}\right)_{\alpha}^{\#}(x)$, let $1<s<p$ and $\frac{1}{t}=\frac{1}{s}-\alpha$. Hölder's inequality with exponent $t$ followed by the Hardy-Sobolev 
inequality (see $[\mathrm{GV}],[\mathrm{S}]$ ) give

$$
\begin{aligned}
\frac{1}{\mu(B)^{1+\alpha}} \int_{B}\left|F_{1}\right| d \mu & \leq \frac{1}{\mu(B)^{\alpha+\frac{1}{t}}}\left(\int_{B}\left|I_{\alpha}(f \chi)\right|^{t} d \mu\right)^{\frac{1}{t}} \\
& \leq\left(\frac{1}{\mu(B)} \int_{\widetilde{B}}|f|^{s} d \mu\right)^{\frac{1}{s}} \leq c\left(\frac{1}{\mu(\widetilde{B})} \int_{\widetilde{B}}|f|^{s} d \mu\right)^{\frac{1}{s}} \\
& \leq c M_{s}(f)(x),
\end{aligned}
$$

where $M_{s}(f)=\left[M\left(|f|^{s}\right)\right]^{\frac{1}{s}}$, and $M$ is the Hardy-Littlewood maximal function. By a known property of the sharp function we have

$$
\left(F_{1}\right)_{\alpha}^{\#}(x) \leq 2 c M_{s}(f)(x)
$$

Now we will estimate $\left(F_{2}\right)_{\alpha}^{\#}(x)$. Observe that

$$
F_{2}(z)-F_{2}\left(x_{0}\right)=\int_{(\widetilde{B})^{c}} f(y)\left[\frac{1}{\delta^{1-\alpha}(z, y)}-\frac{1}{\delta^{1-\alpha}\left(x_{0}, y\right)}\right] d \mu(y) ;
$$

then by $(2.3)$

$$
\int_{B}\left|F_{2}(x)-F_{2}\left(x_{0}\right)\right| d \mu(z) \leq K \int_{B} \delta^{\gamma}\left(z, x_{0}\right)\left(\int_{(\widetilde{B})^{c}} \frac{|f(y)|}{\delta^{1-\alpha+\gamma}\left(x_{0}, y\right)} d \mu(y)\right) d \mu(z) .
$$

To estimate the inner integral we write

$$
\begin{aligned}
\int_{(\widetilde{B})^{c}} & \frac{|f(y)|}{\delta^{1-\alpha+\gamma}\left(x_{0}, y\right)} d \mu(y) \leq \sum_{k=1}^{\infty} \int_{2^{k-1} r \leq \delta\left(x_{0}, y\right)<2^{k} r} \frac{|f(y)|}{\left(2^{k-1} r\right)^{1-\alpha+\gamma}} d \mu(y) \\
\leq & 2^{1-\alpha+\gamma} r^{\alpha-\gamma} \sum_{k=1}^{\infty} \frac{1}{2^{(\gamma-\alpha) k}} \frac{1}{2^{k} r} \int_{B_{2^{k} k_{r}}\left(x_{0}\right)}|f(y)| d \mu(y) \leq c r^{\alpha-\gamma} M f(x) .
\end{aligned}
$$

Using this estimate in (3.2) and a known property of the sharp function we get

$$
\left(F_{2}\right)_{\alpha}^{\#}(x) \leq 2 c M f(x) .
$$

Since $M f(x) \leq M_{s} f(x),(3.1)$ and (3.3), and the fact that the sharp operator is subadditive we have

$$
F_{\alpha}^{\#}(x) \leq c M_{s}(f)(x)
$$

Finally, using the strong type $\frac{p}{s}$ of $M$ we have

$$
\left\|F_{\alpha}^{\#}\right\|_{p} \leq c\|f\|_{p}
$$

This concludes the proof of Theorem 2.1.

Proof of Theorem 2.2. The proof of Theorem 2.2 is similar to that of Theorem 2.1. Set

$$
F-C_{F}=\widetilde{I}_{\alpha}(f \chi)+\widetilde{I}_{\alpha}(f(1-\chi))=F_{1}+F_{2} .
$$

To estimate $\left(F_{1}\right)_{\alpha}^{\#}(x)$, let $c_{B}=-\int_{X}(f \chi) \frac{\psi_{u}(y)}{\delta^{1-\alpha}(u, y)} d \mu(y)$ and $1<s<\frac{1}{\alpha}, \frac{1}{t}=\frac{1}{s}-\alpha$. Then by the same argument used in the proof of Theorem 2.1 we get

$$
\frac{1}{\mu(B)^{1+\alpha}} \int_{B}\left|F_{1}-c_{B}\right| d \mu \leq c M_{s}(f)(x) .
$$


To estimate $\left(F_{2}\right)_{\alpha}^{\#}(x)$, note that

$$
F_{2}(z)-F_{2}\left(x_{0}\right)=\int_{(\widetilde{B})^{c}} f(y)\left[\frac{1}{\delta^{1-\alpha}(z, y)}-\frac{1}{\delta^{1-\alpha}\left(x_{0}, y\right)}\right] d \mu(y)
$$

and therefore as in Theorem 2.1 we have $\left(F_{2}\right)_{\alpha}^{\#}(x) \leq 2 c M f(x)$. The rest of the proof is the same as that of Theorem 2.1.

\section{REFERENCES}

[CDS] P. Cifuentes, J. R. Dorronsoro, and J. Sueiro, Boundary tangential convergence on spaces of homogeneous type, Trans. Amer. Math. Soc. 332 (1992), 331-350. MR 92j:42019

[CS] A. P. Calderón and R. Scott, Sobolev type inequalities for $p>0$, Studia Math. 62 (1978), 75-92. MR 58:7057

[CW] R. Coifman and G. Weiss, Extensions of Hardy spaces and their use in analysis, Bull. Amer. Math. Soc. 83 (1977), 569-645. MR 56:6264

[F] G. B. Folland, Subelliptic estimates and function spaces on nilpotent Lie groups, Arkiv f. Math. 13 (1975), 161-207. MR 58:13215

[GV] A. E. Gatto and S. Vági, Fractional integrals on spaces of homogeneous type, in Analysis and Partial Differential Equations, Cora Sadosky, editor, Marcel Dekker, New York and Basel, 1990. MR 91e: 42032

[MS] R. Macías and C. Segovia, Lipschitz functions on spaces of homogeneous type, Adv. Math. 33 (1979), 257-270. MR 81c:32017a

[NRS] A. Nagel, W. Rudin, and J. Shapiro, Tangential boundary behavior of functions in Dirichlet type spaces, Ann. of Math. 116 (1982), 331-360. MR 84a:31002

[S] E. M. Stein, Harmonic analysis, real-variable methods, orthogonality, and oscillatory integrals, Princeton Univ. Press, Princeton, NJ, 1993. MR 95c:42002

Department of Mathematics, DePaul University, Chicago, Illinois 60614-3504

E-mail address: aegatto@condor.depaul.edu 